\title{
Effects of sleeping position on back pain in physically active seniors: A controlled pilot study
}

\author{
Gustavo Desouzart $^{\mathrm{a}, *}$, Rui Matos ${ }^{\mathrm{a}, \mathrm{b}}$, Filipe Melo ${ }^{\mathrm{c}}$ and Ernesto Filgueiras ${ }^{\mathrm{d}}$ \\ ${ }^{\mathrm{a}}$ Human Movement Research Center, Polytechnic Institute of Leiria, Leiria, Portugal \\ ${ }^{\mathrm{b}}$ School of Education and Social Sciences, Polytechnic Institute of Leiria, Leiria, Portugal \\ ${ }^{\mathrm{c}}$ Faculty of Human Kinetics, the Technical University of Lisbon, Lisbon, Portugal \\ ${ }^{\mathrm{d}}$ LabCom - University of Beira Interio, Covilhã, Portugal
}

Received 28 April 2013

Accepted 6 February 2014

\begin{abstract}
.
BACKGROUND: The increase in life expectancy of elderly population has aroused the interest of different knowledge areas in understanding the variables that are involved in the aging process, linking them to other concepts such as active aging, healthy aging and the bio-psycho-social changes.

OBJECTIVE: This paper presents the results of the first controlled, experimental pilot study that aimed to analyze the relationship between the perception of back pain and the sleeping position adopted by physically active female seniors.

METHODS: Twenty female seniors (mean age 62.70 \pm 3.827 ) participated in this study. The individuals were separated in 2 groups (Experimental and Control Group). For the carrying out of this study, the Visual Analogue Scale (VAS) was used to measure the intensity of back pain in the spine before and after four consecutive weeks an Intervention program. Individuals in the Experimental Group were instructed regarding the recommended way to sleep position (Intervention program) according to the pathological problems or the amount of pain reported.

RESULTS: The Experimental Group $(N=10)$ presented significantly $(p=0.009)$ fewer complaints of back pain after an Intervention program in comparison to individuals who did not receive this type of information (Control Group).
\end{abstract}

Keywords: Back pain, recommended sleeping position, Visual Analogue Scale (VAS), active aging

\section{Introduction}

The increases in life expectancy that occurred in the first half of the 20th century in developed countries were the result of rapid delays in mortality, the access to better housing, sanitation and education, a trend to smaller families, growing incomes, and public health measures has as a principal consequence the appearance of a growing aged population [1]. The

\footnotetext{
*Address for correspondence: Gustavo Desouzart, 25 de Abril street, 72-C, Gândara dos Olivais 2415-600 Leiria, Portugal. Tel.: +351 911973 370; Fax: +351 244092 154; E-mail: gustavodesouzart@gmail.com.
}

older population is one of the fastest growing age cohorts in the United States [2].

Population aging, according to the World Health Organization [(WHO) 1], is "... one of humanity's greatest triumphs and one of our greatest challenges."

The bio-psycho-social changes that seniors undergo determine the importance of promoting a better quality of life, simultaneously with the extension of life expectancy. In what concerns this theme, a new concept of aging emerges, "the active aging".

Active aging has its origins in the WHO [1] and is defined as "...the process of optimizing 
opportunities for health, participation and security in order to enhance quality of life as people age." Maintaining an ideal posture and doing physical exercise are essential to have a healthy body, free of pains, especially those in the spine. An inadequate posture while sleeping and the presence of nervous tension can lead to pain.

Posture is defined in various ways considering the biomechanical alignment and the spatial arrangement of body parts in relation to their segments [3-5]. The risk of back pain is due to a multi-factorial nature, being that poor posture is one of these factors. Poor posture is understood as postures deviating from neutral spinal curvature [6].

Body posture alterations are one of the most frequent problems experienced during advanced age. Stelmach, Populin \& Muller [7] showed that older adults were slower in initiating the movements, which suggests that, with advancing age, the postural stabilization preceding movements may become slower.

This pilot study related concepts such as perception of back pain and / or discomfort with the sleeping position, through a literature review, the application of questionnaires and an intervention in a selected group. Thus, based on these concepts, it is important to know the implications that the sleeping position and a bad posture while sleeping, getting up and lying in bed, can determine on the body dynamics.

Throughout the course of our lives, one third is spent lying down, either resting or sleeping. This period is essential to the musculoskeletal system, as well as to the central nervous system recovery. Gracovetsky [8] considers that the best sleeping position is one that reduces stress, relaxes muscles decreasing their activity, and promotes a better body balance.

The main advantage of sleeping in a supine position, according to Haex [9], is the fact that body weight is distributed over a large surface, resulting in stability being optimized. Most authors suggest that a neutral position of the spine is optimal for spinal health, some suggest a supine position [10].

According to Haex [9], the lateral position is the most adopted sleeping posture, and it is able to support the human spine correctly when both the sleep system and pillow are well conceived: The spinal column is a straight line when projected in a frontal plane, while natural curves (cervical lordosis, thoracic kyphosis, and lumbar lordosis) are maintained. There are no differences between sleeping on the left or on the right side, except the weight of the liver working on the stomach and the lungs when sleeping on the left side. The same author [9] refers that, due to decreased contact surface and the center of gravity being more elevated, a lateral position is an unstable sleeping position, which can be altered by the ideal positioning of the extremities but the use of pillows can be an easy solution for the prevention of torsion of both the pelvic and shoulder girdles; putting a pillow, blanket, or pre shaped pillow between the knees as a spacer will stabilize the most elevated leg in a horizontal position.

The prone sleep position, the lying position facing down, is the most unfavorable posture in relation to back support and, the prone position should be avoided [9-13].

According to Granata and Bennett [14], biomechanical risk factors for low-back disorders include trunk movement and external force, given their relationship with spinal load and stability.

Being the concept of pain broad [15], varying with the personal perception was proposed relating it to active aging in people without a restrictive clinical diagnosis. The main objective of this study was to compare the perception of back pain in the spine of physically active female seniors, aged between 50 and 74 , when adopting different sleeping positions.

\section{Methods}

\subsection{Subjects}

The sample used in the current study was of a nonprobabilistic type and chosen by convenience with a small number of participants to better control the variables applied.

The elderly group analyzed is composed of the participants in the physical activity program 60+, of the Polytechnic Institute of Leiria (IPL) Portugal. Of the 50 elderly participants in this program, only 39 completed the questionnaire proposed. 5 participants were excluded due to answering the questionnaires incorrectly. Initially, 34 seniors responded to the questionnaire, 8 showed grade 0 , and therefore were excluded from the study for not meeting the inclusion criteria, and 6 were male and did not participate in this study. It was composed of 20 participants (female) aged between 55 and 69 (mean $62.70 \pm 3.827$ ). Exclusion criteria were medical problems that can interfere with normal sleep, e.g. intake of sleep medication and antidepressants. All subjects signed an informed consent and the study was approved by the Ethics Committee of IPL. 
The individuals were divided into 2 groups, randomly, the Experimental Group and the Control Group composed of 10 individuals each, integrating them according to the level of pain (5 participants with greater than 5 pain intensity and 5 participants with less than 5 pain intensity in each group).

\subsection{Experimental design}

All the participants that consented to participate in the study and that answered correctly to the abovementioned questionnaires were selected and divided into two groups: Group 1 (experimental), which received instructions about the recommended sleeping position, and group 2 (control), which did not receive any information. There was a great concern that the participants of group 1 did not provide any information to the participants of group 2 .

The second phase of the research project was to provide information to the Experimental Group on the recommended posture while sleeping, how to get up and lie down, and also the amount of pillows and ideal place to use them. The culmination of this phase of the project was the completion of the questionnaire again to all participants on the effects of sleeping position on the back pain in physically active female seniors.

\subsection{Procedure and measures}

It is very difficult to objectively quantify the perception of pain. Pain is always subjective; it is an unpleasant sensory and emotional experience associated with actual or potential tissue damage, or described in terms of such damage $[16,17]$.

For the carrying out of this study, the Visual Analogue Scale (VAS) was used to measure the intensity of Back Pain in the Spine [18-21]. The VAS contains a four-inch long line, which presents the numbers 0 and 10 at each end, respectively. The 0 indicates no pain and 10 the worst pain imaginable and each individual is asked to identify, on this scale, the intensity of their pain.

According to the measurement instrument, this form was applied individually.

The duration of the intervention with the Experimental Group was four consecutive weeks. In the first week, some questionnaires were carried out: a) the questionnaires of personal identification; $b$ ) measurement of pain and/or discomfort at the moment of the response to the questionnaire; and c) an informal questionnaire for the lying down/getting up and sleeping position.

In the 1st week, the individuals selected for the experimental group were given the recommendations and indications of the ideal sleeping position, as well as the ideal quantity, height and place for the positioning of the pillow when sleeping.

According to the indications of authors such as Gracovetsky [8]; Haex [9]; Tetley [22]; Edwards [23] and Oliver and Middleditch [24], individuals in the experimental group were instructed to change their initial sleeping position according to the pathological problems or the amount of pain reported. Figure 1

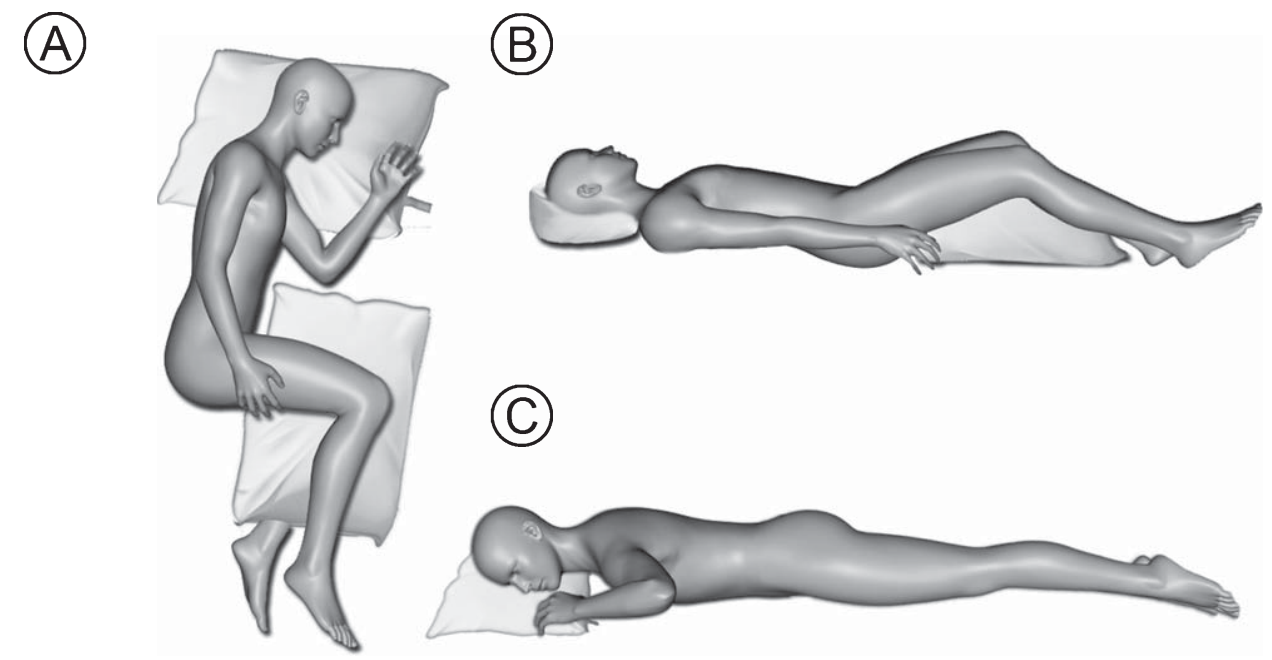

Fig. 1. Recommended sleeping positions and pillow orientation (A - lateral position and B - supine sleep position). Not recommended prone sleep position ( $\mathrm{C}$ - prone sleep position). 
shows the two types of recommendations and, those who complained of dorsal or low back pain, were nominated for the posture A and those who possessed cervical complaints were oriented for posture B. Those who possessed the habit of sleeping in Prone Position (Posture C) were instructed to assume the position A or B, given that some authors associate this with the attitude that should be avoided [9-13].

For the experimental group to adopt new patterns of postural behavior while sleeping, 4 weeks of adaptation were given. At the end of the 4 th week, the questionnaires were carried out again. The seniors that received indications also answered an informal form questionnaire concerning the perception of the changes that occurred after the experimental period.

Data were analysed using a statistical analysis program - Statistical Package for the Social Sciences (SPSS), version 19.0. Wilcoxon test for Non-parametric statistics were used (comparing the same group at different times), setting the level of significance at $p \leq 0.05$.

\section{Results}

Concerning the Experimental Group (participants who received indications of the recommended sleeping position), 9 (90\%) reported a decrease in pain (improvement in well-being) after the carrying out of the given instructions and only $1(10 \%)$ indicated that the pain was still present but stable. We compared the final results with the initial results of the two groups (control and experimental), using the Wilcoxon nonparametric test. The samples are relatively small, so we need a nonparametric procedure. Since we are comparing two related, or paired, samples, we will use the wilcoxon signed ranks test [25].
Table 1

Perception of pain in the Initial and Final VAS

\begin{tabular}{llcc}
\hline Groups & & Initial VAS & Final VAS \\
\hline Experimental & Average pain & $5.40 \pm 2.01$ & $3.00 \pm 1.63$ \\
Group $(n=10)$ & Minimum & 2 & 0 \\
& Maximum & 8 & 6 \\
Control & Average pain & $4.30 \pm 2.36$ & $3.90 \pm 3.21$ \\
Group $(n=10)$ & Minimum & 1 & 0 \\
& Maximum & 8 & 9 \\
\hline
\end{tabular}

Before following the indications, the 10 participants of the Experimental Group had, in average, a perceived level of pain of $5.40 \pm 2.01$ (on a scale of 0 to 10), with a referred minimum of 2 and a maximum of 8 . After the intervention, the seniors that followed the indications about the recommended sleeping posture for 4 consecutive weeks, verified that the perception of pain decreased significantly to an average of $3.00 \pm 1.63(p=0.009)$, with a minimum pain level of 0 and a maximum pain level of 6 .

The remaining 10 participants (Control Group) that did not receive indications had a lower decrease of the average level of pain perception compared to the Experimental Group. They passed the pain perception's initial average level of 4.30 to 3.90 (non-significant decrease $-p=0.472$ ). Concerning the maximum level of pain, it increased 1 point, reaching level 9 (Table 1). The relation between the perception of pain in the initial and final evaluation time, according to the VAS, showed a greater difference in the responses of the participants who received instructions concerning the recommended sleeping position (Experimental Group), with an average of less 2.40 of pain $(p \leq 0.05)$. The participants who did not receive any instruction (Control Group), showed a decrease in the average pain value of 0.40 (Fig. 2).

These final indications were used to start the following discussion, according to the main objectives of this study).

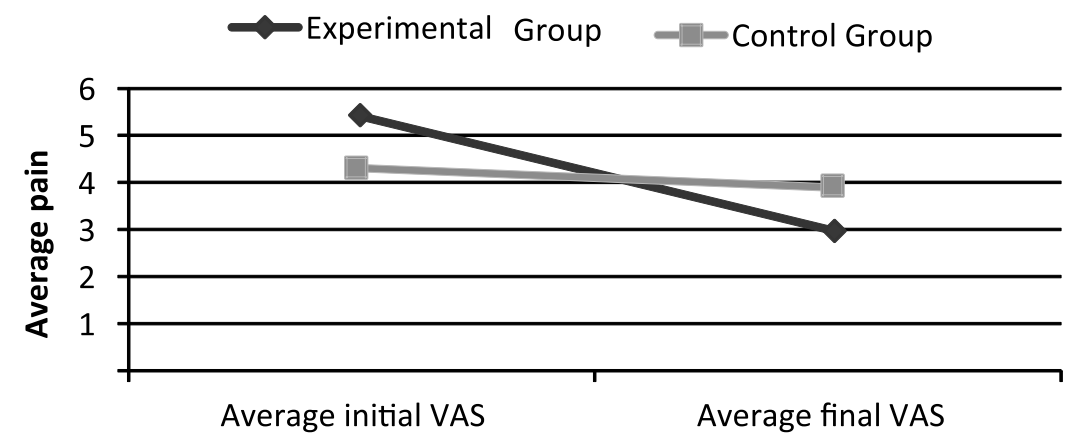

Fig. 2. Experimental and control group average data concerning pain in the initial and final VAS. 


\section{Discussion and conclusions}

The indications provided to the participants were related to having a recommended posture in the sleeping position, taking into account the loads and forces that influence the human body at all moments in life.

Regarding the 10 participants of the Experimental Group, as previously stated, 9 (90\%) indicated a significant decrease in the level of pain after 4 consecutive weeks ( $p=0.009)$, which enables the validation of the hypothesis proposed in the beginning of the study.

In the study's initial evaluation period, the Experimental Group had an average level of 5.40, on a scale of 0 to 10 , of the perception of pain (according to the VAS). In the final evaluation period, the perception of pain decreased to an average of 3.00. This difference of less 2.40 in the pain level indicates that the positive difference was due to the information provided (Fig. 2).

The results observed in this study concerning the perception of pain associated with the posture adopted while sleeping, showed a statistically significant decrease for the Experimental Group $(p=0.009)$ and not a statistically significant result for the Control Group ( $p=0.472$ ) [Fig. 2].

This fact can confirm that the reduction of the level of pain is greater in the group that received information concerning the recommended sleeping position. Initially this group had more pain, in average, than the control group. At the end of the study, the Experimental Group presented, in average, a lower level of pain.

Given the progressive aging of the population, which occurs mainly in the developed countries, societies today have a new concern and a new way to face this phenomenon. This fact can be associated with the creation of a new concept, the active aging, which intends to enable seniors to age in an active, independent and successful way.

The aim of active aging is to increase healthy life expectancy and quality of life for people who are aging. Reducing levels of pain, maintaining a good posture when sleeping, and the autonomy and independence during the aging process enables the person to be healthier, and this is a key goal for society.

The longevity that is gained due to the progress of science and improvement of quality of life promotes the opportunity for seniors to enjoy more of their free time, compared to the working time and stimulates the development of preventive methods and techniques concerning the preservation of a healthy body, such as sleeping in a proper position.
The issue addressed in this study about the influence of the sleeping position on the perception of pain and/or discomfort in the spine in physically active female seniors, reveals a decrease in the level of pain and/or discomfort in the spine of the participants that received information about the recommended sleeping position, associated with the vertebral alignment and the musculoskeletal adaptations in the spine.

It may be concluded that the indication of the ideal way to lie down, which corresponds to a recommended sleeping posture with the ideal position to place the pillows, as well as the ideal way to get up (Experimental Group), is an added value for the prevention and decrease of the pain and/or discomfort in the spine in physically active seniors.

\section{Limitations}

Generally, programs related to exercise and health in Portugal, such as the physical activity program $60+$, have more female than male participants and a reduced number of participants. Thus, it was not possible to use a homogeneous sample and a larger number of participants. The four weeks may not have been sufficient to create habits in participants, however, and because of the time limitations of this study, it was not possible to have a longer time.

We were unable to follow participants in the experimental group during sleep and results are based on the statements of the participants who reported having changed its initial stance before bed. Because this study only had to assess whether there were differences after a program of recommendations, it is believed that the objectives were fulfilled.

A new study is ongoing and will be used in a young population with diagnosed problems. The sleeping habits will be monitored through observation and video, systemic use of pressure sensors and motion and time adaption will be provided over 12 weeks.

According to Huang et al. [26], the multimodal approach to human sleeping posture classification, using pressure sensor array and video camera as complementary modalities for feature extraction and classification, indicate that the combination of both modalities can lead to a better performance of the posture classification.

A larger number of participants may allow better observation of the changes proposed and the results obtained. 
Will be used in a future study to apply a methodology for systematic observations of the posture while sleeping through digital video recording [27, 28].

\section{Acknowledgments}

This study was supported by the Human Movement Research Center of the Polytechnic Institute of Leiria (IPL) Portugal.

The authors would like to acknowledge the IPL and in particular it's School of Education and Social Sciences and School of Health Sciences. The authors also thank the program $60+$ of IPL for assisting in the sample collection process and the research assistants for their help during the data collection phase. The authors want to thank the InEnglish workers, in particular Paula Santos and Nelia Diogo for their excellent contribution to the article literature review.

We would also like to thank the study participants for their contribution of time and effort to the research.

\section{References}

[1] World Health Organization. Active Ageing: A Policy Framework. 2002, pp. 6-12. Retrieved May 4, 2010, Available from: http://whqlibdoc.who.int/hq/2002/ who_nmh_nph_02.8.pdf.

[2] Kampfe C, Wadsworth J, Mamboleo G, Schonbrun S. Aging, disability, and employment. WORK: A Journal of Prevention, Assessment \& Rehabilitation. IOS Press 2008;31:337-44.

[3] Rohmert W, Mainzer J. Influence and assessment methods for evaluating body postures. In: Corlett N,Wilson J, Manenica I, eds. The ergonomics of working postures. London: Taylor and Francis; 1986, pp. 183-217.

[4] Silva A, Punt T, Sharples P, Vilas-Boas J, Johnson M. Head posture assessment for patients with neck pain: Is it useful? International Journal of Therapy and Rehabilitation 2009;16(1):43-53.

[5] Vieira E, Kumar S. Working postures: A literature review. Journal of Occupational Rehabilitation 2004;14(2):143-59.

[6] Geldhof E, Clercq D, Bourdeaudhuij I, Cardon G. Classroom postures of 8-12 year old children. Ergonomics 2007;50(10):1571-81.

[7] Stelmach G, Populin L, Muller F. Postural muscle onset and voluntary movement in the elderly. Neuroscience Letters 1990;117(1-2):188-93.

[8] Gracovetsky S. The resting spine: A conceptual approach to the avoidance of spinal reinjury during rest. Physical Therapy 1987;67(4):549-53.

[9] Haex B. Back and Bed: Ergonomic Aspects of Sleeping. Boca Raton: CRC Press; 2005.
[10] Gordon S, Grimmer K, Trott P. Sleep position, age, gender, sleep quality and waking cervico-thoracic symptoms. The Internet Journal of Allied Health Sciences and Practice. 2007;5(1). Retrieved Jan. 11, 2011, Available from: http:// ijahsp.nova.edu/articles/vol5num1/gordon2.pdf.

[11] Bland J. Disorders of the Cervical Spine. Philadelphia: Saunders; 1987.

[12] Grieve G. Common Vertebral Joint Problems. 2nd ed. Churchill Livingstone, Edinburgh; 1988.

[13] Kramer J. Intervertebral Disk Diseases: Causes, Diagnosis, Treatment and Prophylaxis. 2nded. New York: Thieme Medical Publishers; 1990.

[14] Granata K, Bennett B. Low-back biomechanics and static stability during isometric pushing. Human Factors 2005;47(3):536-49.

[15] McCaffery M, Pasero C. Pain ratings: The fifth vital sign. American Journal of Nursing 1997;97(2):15-6.

[16] International Association For The Study Of Pain. Classification of chronic pain. 2nded., Seattle, IASP Press; 1994.

[17] Oberlander T, Symons F. Pain in Children \& Adults with Developmental Disabilities. Baltimore: Paul H. Brookes Publishing Co.; 2006, p. 246.

[18] Menéndez C, Amick III B, Jenkins M, Caroom C, Robertson M, Gerr F, Moore S, Harrist R, Katz J. A validation study comparing two self-reported upper extremity symptom surveys with clinical examinations for upper extremity musculoskeletal disorders. WORK: A Journal of Prevention, Assessment \& Rehabilitation IOS Press 2012;43:293-302.

[19] Bieri D, Reeve R, Champion G. The faces pain scale for the self-assessment of the severity of pain experienced by children: Development, initial validation and preliminary investigation for ration scale properties. Pain 1990;41: 139-50.

[20] Ferreira-Valente M, Pais-Ribeiro J, Jensen M. Validity of four pain intensity rating scales. Pain 2011;152:2399-404.

[21] Price D, Mcgrath P, Rafii A, Buckingham B. The validation of visual analogue scales as ratio scale measures for chronic and experimental pain. Pain 1983;17(1):45-56.

[22] Tetley M. Instinctive sleeping and resting postures: An anthropological and zoological approach to treatment of low back and joint pain. BMJ 2000;321:1116-618.

[23] Edwards J. The importance of postural habits in perpetuating myofascial trigger point pain. Acupuncture in Medicine 2005;23(2).

[24] Oliver J, Middleditch A. Functional anatomy of the spine. Butterworth-Heinemann Ltd.; 1991.

[25] Corder G, Foremam D. Nonparametric Statistics for NonStatisticians: A Step-by-Step Approach. New Jersey: John Wiley \& Sons. 2009.

[26] Huang W, Aung A, Foo S, Biswas J. Multimodal Sleeping Posture Classification. International Conference on Pattern Recognition. IEEE; 2010, pp. 4336-9.

[27] Rebelo F, Filgueiras E, Soares M. Behavior Video: A Methodology and Tool to Measure the Human Behavior: Examples in Product Evaluation. Handbook of Human Factors and Ergonomics in Consumer Product Design: Methods and Techniques. CRC Press, Taylor \& Francis Group. 2011.

[28] Filgueiras E, Rebelo F, Moreira da Silva F. Support of the upper limbs of office workers during a daily work. WORK: A Journal of Prevention, Assessment \& Rehabilitation. IOS Press. 2012. 